\title{
Discovery of a Novel 2,6-Disubstituted Glucosamine Series of Potent and Selective Hexokinase 2 Inhibitors
}

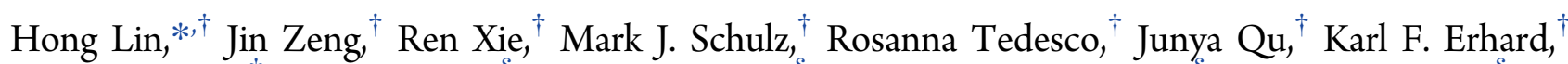
James F. Mack, ${ }^{\dagger}$ Kaushik Raha, ${ }^{\S}$ Alan R. Rendina, ${ }^{\S}$ Lawrence M. Szewczuk, ${ }^{\S}$ Patricia M. Kratz, ${ }^{\S}$ Anthony J. Jurewicz, ${ }^{\S}$ Ted Cecconie, ${ }^{\S}$ Stan Martens, ${ }^{\S}$ Patrick J. McDevitt, ${ }^{\S}$ John D. Martin, ${ }^{\S}$ Stephenie B. Chen, ${ }^{\S}$ Yong Jiang, ${ }^{\S}$ Leng Nickels, ${ }^{\S}$ Benjamin J. Schwartz, ${ }^{\S}$ Angela Smallwood, ${ }^{\S}$ Baoguang Zhao, ${ }^{\S}$ Nino Campobasso, ${ }^{8}$ Yanqiu Qian, ${ }^{\S}$ Jacques Briand, ${ }^{\S}$ Cynthia M. Rominger, Catherine Oleykowski, ${ }^{\ddagger}$ Mary Ann Hardwicke, ${ }^{\ddagger}$ and Juan I. Luengo

${ }^{\dagger}$ Cancer Metabolism Chemistry; ${ }^{\ddagger}$ Cancer Metabolism Biology; and ${ }^{\S}$ Platform Technology \& Sciences, GlaxoSmithKline, 1250 South Collegeville Road, Collegeville, Pennsylvania 19426-0989, United States

\section{Supporting Information}

ABSTRACT: A novel series of potent and selective hexokinase 2 (HK2) inhibitors, 2,6-disubstituted glucosamines, has been identified based on HTS hits, exemplified by compound 1. Inhibitor-bound crystal structures revealed that the HK2 enzyme could adopt an "induced-fit" conformation. The SAR study led to the identification of potent $\mathrm{HK} 2$ inhibitors, such as compound $\mathbf{3 4}$ with greater than 100-fold selectivity over HK1. Compound $\mathbf{2 5}$ inhibits in situ glycolysis in a UM-UC-3 bladder tumor cell line via ${ }^{13} \mathrm{CNMR}$ measurement of $\left[3-{ }^{13} \mathrm{C}\right]$ lactate produced from $\left[1,6-{ }^{13} \mathrm{C}_{2}\right]$ glucose added to the cell culture.

KEYWORDS: Hexokinase 2 inhibitor, crystal structure, structure-activity relationship selectivity
$\mathrm{I}_{\mathrm{ap}}^{\mathrm{n}}$ nhibition of glycolysis has been considered as a therapeutic approach for anticancer treatment for decades. ${ }^{1}$ As the first enzyme in the glycolytic pathway, hexokinase-2 (HK2) plays two pivotal roles in cancer. HK2 catalyzes the first functionally irreversible reaction in the glycolytic pathway to form glucose6-phosphate (G6P) from glucose and ATP. It drives the "Warburg effect" by committing glucose to enter both the glycolytic and pentose phosphate pathways. Second, HK2 stabilizes the Mitochondrial Outer Membrane (MOM) via interaction with the voltage-dependent anion channel (VDAC) to prevent apoptosis. ${ }^{2}$ The HK2 gene is amplified and HK2 RNA is overexpressed in tumors. ${ }^{3}$ Epigenetic changes such as HK2 DNA demethylation have been reported as well. The most profound changes in HK2 expression have been observed in liver, kidney, endometrial, and pancreatic cancers. ${ }^{4} \mathrm{HK} 2$ expression is regulated by many somatic stimuli: HIF1- $\alpha$, growth factors, c-MYC, and p53 mutations. Akt stimulates HK2 association with mitochondria to promote mitochondrial integrity and prevent apoptosis. HK2 expression correlates with tumor stage and a worse prognosis. Studies with HK2 knockdowns using siRNA or shRNA both in vitro and in vivo have shown inhibition of tumor growth. ${ }^{5,6}$

Recently, Hay and his collaborators provided compelling evidence for the role of $\mathrm{HK} 2$ in tumorigenesis and maintenance, as well as metabolic insights via Hk2 depletion using a genetic mouse model of nonsmall cell lung carcinoma (NSCLC) induced by expression of activated KRAS (KRASLA2) and a mouse model of breast cancer induced by expression of activated ERBB2/Neu (MMTV-neu). ${ }^{7}$ They also showed that global $\mathrm{Hk} 2$ ablation in adult mice was welltolerated without significant physiological consequences.

While HK2 is a potential target for cancer treatment, it has been considered intractable for the past 50 years due to its extremely polar active site, the complexity of its protein functions, and the uncertainty associated with finding a $\mathrm{HK} 2$ selective inhibitor over the housekeeping HK1 isozyme. Encouraged by the strong rationale, we initiated research to identify HK2 selective small-molecule inhibitors for potential cancer treatment or in combination with existing drugs to sensitize chemotherapy and targeted therapy.

Compound 1 was one of the glucosamine derivatives identified from the high throughput screen (Supporting Information) with purified HK2, and we first synthesized a variety of C-2 amides to examine potency and HK2 vs $\mathrm{HK} 1$ selectivity. ${ }^{8}$ As shown in Table 1 , compound $\mathbf{1}$ is weakly active against $\mathrm{HK} 2$, but has no selectivity over HK1. Using a continuous coupled assay (formation of G6P coupled to G6P dehydrogenase), compound $\mathbf{1}$ was found to be competitive with glucose $\left(K_{\mathrm{i}}=2.9 \pm 0.33 \mu \mathrm{M}\right)$ and noncompetitive versus MgATP (Supporting Information). In a separate double inhibition study (continuous coupled assay for ADP formation with pyruvate kinase and lactate dehydrogenase), compound 1

Received: May 29, 2015

Accepted: December 27, 2015

Published: December 28, 2015 
Table 1. SAR of C-2 Substituted Glucosamines<smiles>[R]C(=O)NC1C([R6])OC(CO)C(O)C1O</smiles>

\begin{tabular}{|c|c|c|c|c|}
\hline \multirow{2}{*}{$\#$} & \multirow{2}{*}{ R1 } & \multirow{2}{*}{$\mathbf{R 2}$} & \multicolumn{2}{|c|}{ Enzyme $\mathrm{IC}_{50}(\mu \mathrm{M})^{\mathrm{a}}$} \\
\hline & & & HK2 & HK1 \\
\hline 1 & & $\mathbf{O H}$ & 6.3 & 2.0 \\
\hline 2 & & $\mathbf{O H}$ & 16 & 13 \\
\hline 3 & & $\mathbf{O H}$ & 3.2 & 0.79 \\
\hline 4 & & $\mathbf{O H}$ & 0.79 & 0.040 \\
\hline 5 & & $\mathbf{O H}$ & 16 & 160 \\
\hline 6 & & OH & 2.0 & 2.0 \\
\hline 7 & & OMe & $>400$ & $>\mathbf{2 0 0}$ \\
\hline 8 & & H,H & $>400$ & $>200$ \\
\hline
\end{tabular}

${ }^{a} \mathrm{IC}_{50}$ values given are means of at least 2 experiments.

was also shown to bind simultaneously with G6P (Supporting Information). From structural studies with HK1, G6P is known to bind separately from glucose in a nearby "allosteric" site with the pyranose ring at the putative position of the ATP-bound $\mathrm{Mg}^{2+}$ cation. ${ }^{9}$ Further modifications of the C-2 amides indicated that bulky substitutions at the meta-positions of the benzene ring improve $\mathrm{HK} 2$ potency (see 3 and 4 ). However, such modifications impacted HK1 potency more; for example, compound 4 was a very potent HK1 inhibitor with an $\mathrm{IC}_{50}$ of $40 \mathrm{nM}$. On the other hand, a bulky aliphatic amide as in compound 5 appeared to enhance HK2 selectivity $\left(\mathrm{IC}_{50}=16\right.$ and $160 \mu \mathrm{M}$, respectively, for HK2 and HK1), but suffered from weak potency. Compound 6 with a 3,5-dinitrobenzamide is equally active against $\mathrm{HK} 2$ and $\mathrm{HK} 1$ with an $\mathrm{IC}_{50}$ value of 2.0 $\mu \mathrm{M}$.

We also examined the importance of the C1-hydroxy group for inhibitor potency. Unfortunately, both the 1-methoxy analog (7) and the 1 -deoxy analog $(8)^{10}$ were inactive.

To understand the binding of these inhibitors with HK2, we conducted crystallography studies, and obtained the very first ligand bound human HK2 cocrystal structure at $2.76 \AA$ with compound 1 and glucose-6-phosphate (G6P). ${ }^{11}$ The structure is consistent with the double inhibition kinetic study, which showed G6P and compound 1 could bind simultaneously to $\mathrm{HK} 2$. Both $\mathrm{N}$ - and C-terminal domains show compound $\mathbf{1}$ at their corresponding active sites, with the glucosamine ring placed in the glucose-binding pocket (Figure 1), which is consistent with the glucose competitive mode of action of these compounds. All donor-acceptor interactions of the hydroxyl groups of glucose with $\mathrm{HK} 2$ are also retained in the interaction of compound 1 with HK2. Since the 1-hydroxyl group forms a tight hydrogen bonding network with a water molecule and the side chains of Gln739 and Glu742, it is not surprising that the 1-methoxy (7) and 1-deoxy (8) modifications are not tolerated.

The cocrystal structure of HK2 with compound 1 reveals that the enzyme binding site is highly flexible. Comparison with the HK2-glucose cocrystal structure $(2 \mathrm{NZT})^{12}$ shows that, in both crystals (Figure 1A), the glucose ring binds tightly to the big lobe of the enzyme via hydrogen bonds to Glu708, Gln739, and Glu742. But for compound 1, the active-site loop (residues 616-633, colored red in Figure 1), which contains the catalytic Lys621, is unable to fully close over the pyranose ring, as it is displaced outward by $3 \AA$ due to steric clash with the C-2 amide side chain. This prevents the enzyme from phosphorylating the C-6 hydroxyl group of compound 1. Because of such displacement, the narrow pocket extending from the C-2 equatorial position of the pyranose ring is considerably widened and can now easily accommodate bulkier amide substituents, a clear example of enzyme "induced fit" by a ligand. This wider pocket is lined with side chains from Pro605, Lys618, Lys738, and Gln739 and is highly lipophilic, which explains the increased potency of the biphenyl-derivative 4 (Table 1). A hydrogen bond between the C-2 amide carbonyl and the Thr620 side chain helps to direct the C-2 substituent into this lipophilic pocket. The structure also suggests that the extension of the molecule into the G6P binding site through derivatization at the C- 6 position could be a way to improve potency of the glucosamine series. Since three oxygen atoms of
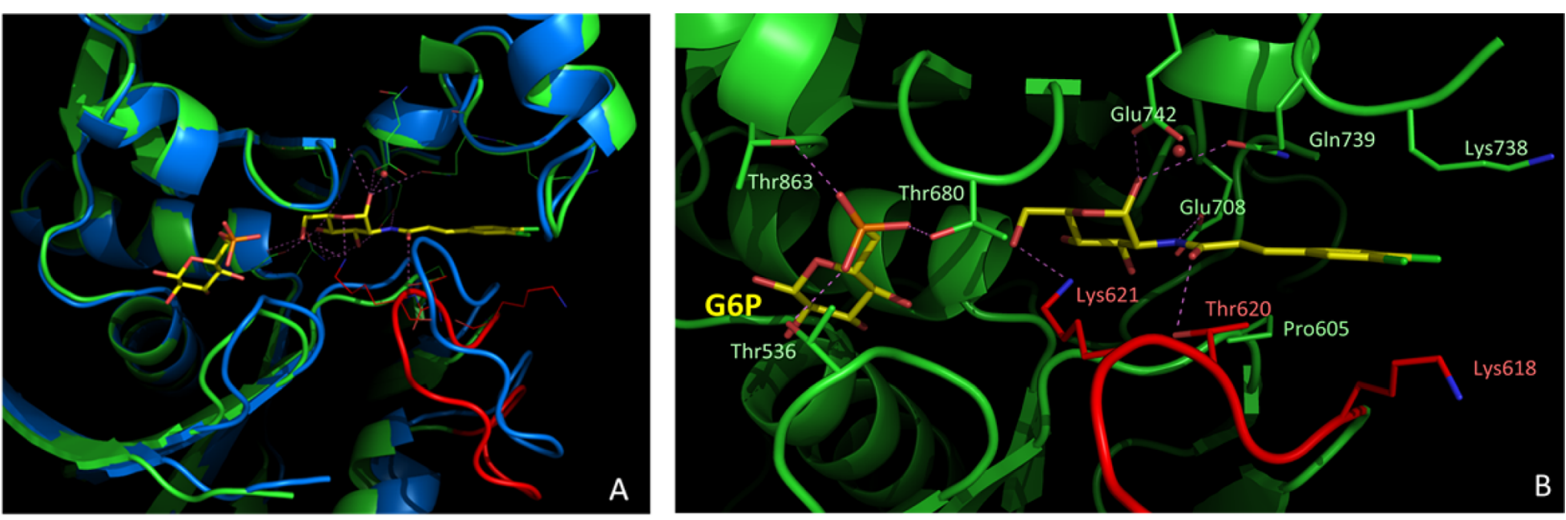

Figure 1. (A) Compound 1 in HK2 with G6P overlaid with 2NZT; (B) Compound 1 and G6P interactions with HK2 in the C-terminal catalytic pocket. 
the phosphate of G6P form H-bond interactions with Thr536, Thr680, and Thr863, a polar group to mimic one of the $\mathrm{H}$ bond interactions might be important.

Based on this hypothesis, we set out to modify the 6-position as depicted in Scheme 1, in which the 1-hydroxy of compound

Scheme 1. Synthesis of 2,6-Disubstituted Glucosamines

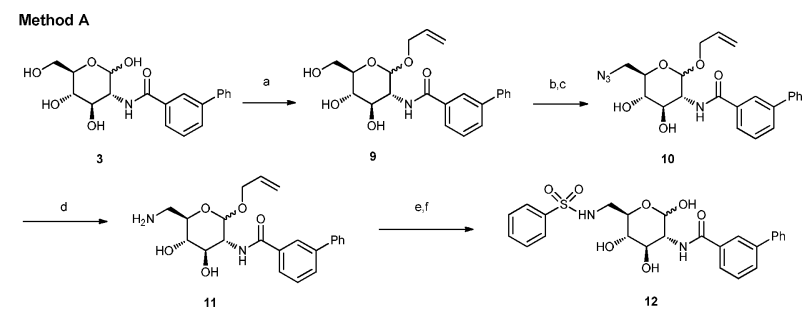

Method B
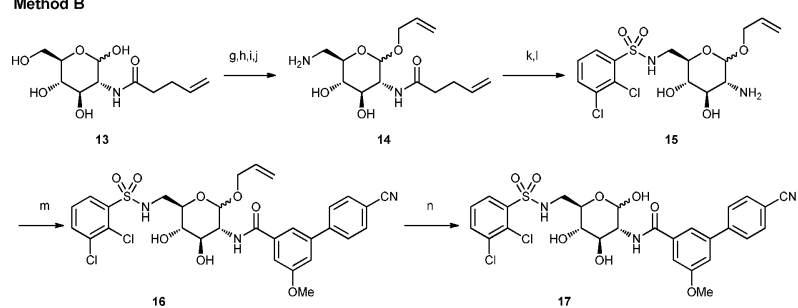

Conditions and Reagents: (a) allyl alcohol, TsOH$\cdot \mathrm{H}_{2} \mathrm{O}, 110{ }^{\circ} \mathrm{C}, 72 \mathrm{~h}$, 93\%; (b) $\mathrm{TsCl}$, py, rt, $3 \mathrm{~h}$; (c) $\mathrm{NaN}_{3}, \mathrm{DMF}, 90{ }^{\circ} \mathrm{C}, 17 \mathrm{~h}$; 52\%; (d) $\mathrm{Ph}_{3} \mathrm{P}, \mathrm{H}_{2} \mathrm{O}$, THF, rt, $72 \mathrm{~h}, 43 \%$; (e) benzensulfonyl chloride, py, rt, 20 h; (f) $\mathrm{PdCl}_{2}, \mathrm{MeOH}, \mathrm{rt}, 4$ h; $19 \%$; (g) allyl alcohol, TsOH $\cdot \mathrm{H}_{2} \mathrm{O}, 110$ ${ }^{\circ} \mathrm{C}, 2 \mathrm{~h}$; (h) TsCl, py, $0{ }^{\circ} \mathrm{C}, 6 \mathrm{~h}, 32 \%$; (i) $\mathrm{NaN}_{3}$, DMF, $80{ }^{\circ} \mathrm{C}, 24 \mathrm{~h}$; (j) $\mathrm{Ph}_{3} \mathrm{P}, \mathrm{H}_{2} \mathrm{O}$, THF, rt, $24 \mathrm{~h}$; (k) 2,3-dichlorobenzensulfonyl chloride, py, rt, $24 \mathrm{~h}, 30 \%$; (l) iodine, THF $/ \mathrm{H}_{2} \mathrm{O} 1: 1, \mathrm{rt}, 2 \mathrm{~h}$; then solid sodium thiosulfate/sodium carbonate; (m) 4'-cyano-5-methoxy-[1,1'-biphenyl]-3-carboxylic acid, 2-(1H-benzotriazol-1-yl)-1,1,3,3-tetramethyluronium hexafluorophosphate (HBTU), diisopropylethylamine, $\mathrm{CH}_{2} \mathrm{Cl}_{2}$, rt, 24 h, 26\%; (n) $\mathrm{PdCl}_{2}, \mathrm{MeOH}, \mathrm{rt}, 24$ h; 46\%.

4 was protected with an allyl group in a reaction catalyzed by $\mathrm{TsOH}$ hydrate or, alternatively, by $\mathrm{BF}_{3} \cdot \mathrm{Et}_{2} \mathrm{O}$ at lower temperature $\left(80^{\circ} \mathrm{C}\right)$ and with shorter reaction time $(18 \mathrm{~h})$. The 6-hydroxy of compound 9 was then converted to the tosylate, which was treated with sodium azide in DMF to form compound 10. Reduction of the azide to a primary amine $\mathbf{1 1}$ with $\mathrm{Ph}_{3} \mathrm{P}$ in $\mathrm{THF} / \mathrm{H}_{2} \mathrm{O}$ provided a key intermediate for $\mathrm{C} 6$ elaboration. As an example, sulfonamide 12 was formed by slow addition of benzenesulfonyl chloride to a solution of compound 11 in pyridine, followed by a Pd-mediated allyl deprotection in $\mathrm{MeOH}$ and reverse phase HPLC purification.

In order to develop a robust synthetic route to modify the 2position of the 2,6-disubstituted glucosamine series, we introduced pent-4-enamide at the beginning to give compound 13. ${ }^{13,14}$ It could be deprotected under mild conditions later in the synthetic scheme. As depicted in Scheme 1, Method B, using the same transformations, the 6-hydroxy of compound $\mathbf{1 3}$ was converted to amine 14. Treatment of 2,3-dichlorobenzenesulfonyl chloride with $\mathbf{1 4}$ followed by deprotection of the pent-4-enamide with iodine afforded a second useful intermediate $\mathbf{1 5}$ for 2-position modification. As an example, compound 15 was converted to amide 16 via a coupling reaction with $4^{\prime}$-cyano-5-methoxy-[1,1'-biphenyl]-3-carboxylic $\mathrm{acid}^{15}$ in the presence of HBTU and DIEA, followed by a Pdmediated deprotection of the allyl group to give the final compound 17 after reverse phase HPLC purification.
Various functional groups at the 6-position, including sulfonamide, amide, and urea, are tolerated (Supporting Information Table 1). Among them, the 6-sulfonamide (compound 18) is particularly promising with improved HK2 inhibitory activity.

Given how much has been known about $\mathrm{HK} 2 / \mathrm{HK} 1$ substrate binding pockets and the fact that they have been considered as intractable targets for the last 50 years, it is remarkable that these dramatic modifications to glucose are tolerated and result in increased inhibition of both $\mathrm{HK} 2 / \mathrm{HK} 1$ enzymatic activities. We therefore pursued this approach together with X-ray crystallography to understand the structural biology of HK2.

Our hypothesis to improve potency against $\mathrm{HK} 2$ by modification of the 6-position of the glucosamine series was further validated with additional 6-sulfonamide analogs. As demonstrated in Table 2, ortho-chloride (23) improves HK2

Table 2. SAR of 2-Amido-6-benzenesulfonamide glucosamines $^{a}$

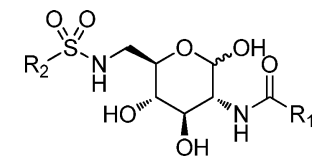

(

${ }^{a} \mathrm{IC}_{50}$ values given are means of at least 2 experiments 
and HK1 potency by 8 - to 10 -fold, respectively, compared to the parent benzenesulfonamide 12 , while 2,3-dichlorobenzenesulfonamide 25 is the first potent $\mathrm{HK} 2$ inhibitor with an $\mathrm{IC}_{50}$ value less than $100 \mathrm{nM}\left(\mathrm{HK} 2 \mathrm{IC}_{50}=25 \mathrm{nM}\right.$, HK1 $\mathrm{IC}_{50}=32$ $\mathrm{nM})$. Heterocyclic sulfonamides are also equally potent, exemplified by 4-chloropyridine-3-sulfonamide (26), compared to its corresponding chlorobenzenesulfonamide 23 .

Some polar substitutions were introduced into the benzensulfonamide to potentially mimic $\mathrm{H}$-bond interactions between the protein and G6P, since the 6-sulfonamides were designed to extend into the polar G6P binding pocket. As an example, a para-nitrile increases the potency of compound 27 against $\mathrm{HK} 1$ by 20 -fold to $7.9 \mathrm{nM}$, while the increase of potency against $\mathrm{HK} 2$ is less profound, only 6-fold.

Interestingly, a meta-carboxylic acid also increases both $\mathrm{HK} 2$ and HK1 potency by 16 -fold and 6-fold (compound 28 versus 12), respectively. The acid-protein interactions appear to be specific, since compound 29, bearing an ester group is far less potent than its corresponding acid $\mathbf{3 0}$. Compound $\mathbf{3 0}$ is 63 -fold more potent against $\mathrm{HK} 2\left(\mathrm{IC}_{50}=10 \mathrm{nM}\right)$, and 80 -fold more potent against $\mathrm{HK} 1\left(\mathrm{IC}_{50}=20 \mathrm{nM}\right)$.

In addition, consistent with the SAR that bulky amides increase HK1 inhibitory potency in the monosubstituted 2glucosamine chemical series, exemplified in Table 1 by compound 5, it is not surprising that compound 17 is very potent against $\mathrm{HK} 1$, but $\mathrm{HK} 2$ potency remains the same as that of compound 25 .

Throughout the course of optimization, we have been seeking the "clues" in SAR to improve HK2 vs HK1 selectivity even though the sequences of these two proteins are $72 \%$ identical and $84 \%$ similar, and the glucose binding sites are well conserved among all hexokinases. ${ }^{16}$ Careful SAR analysis revealed consistent trends for HK2 selectivity: (a) 2,3disubstituted sulfonamides at the 6-position; (b) less bulky amides at the 2-position; and (c) sulfonamide at the 2-position. Combination of any two of the elements could afford a compound as selective as 500 -fold against $\mathrm{HK} 2$ versus $\mathrm{HK} 1$, such as compound 36. This has been proven to be a tractable SAR to yield selective HK2 inhibitors exemplified by compounds 31-36 (Table 3 and Supporting Information Figure 5). Importantly, most selective compounds are also very potent; for example, 2,6-bis-sulfonamide 34 has a HK2 potency of $7.9 \mathrm{nM}$ while its $\mathrm{HK} 1$ potency is only $1.0 \mu \mathrm{M}$. Therefore, it has 125 -fold selectivity against HK2 versus HK1.

With various tool molecules in hand, we successfully obtained some critical cocrystal structures to reveal the binding modes of these 2,6-disubstituted glucosamines. As shown in Figure 2, the cocrystal structure of compound $\mathbf{3 0}$ in $\mathrm{HK} 2,{ }^{17}$ a dimer with 4 molecules of ligands in both $\mathrm{N}$ - and C-terminal catalytic pockets, indeed supports our hypothesis that substitution at the 6-position can extend into the G6P pocket and increase potency. Similar to compound 1, compound $\mathbf{3 0}$ also forms H-bond interactions with Gln742 and Glu708. However, it does not engage Lys621 because the catalytic loop (aa616-633) is no longer in proximity, since the pocket is even more open. The overlay (Figure 2B) of both cocrystal structures reveals: (1) the carboxylic acid group of compound 30 mimics the phosphate of G6P to form similar $\mathrm{H}$-bond interactions with Thr680 and Thr863 but not Thr536; (2) the catalytic loop (aa616-633, salmon and red loops on the right) and the other loop that interacts with G6P (aa533-538, salmon and red loops on the left) are wide open to accommodate the
Table 3. HK2 Selective Glucosamine Derivatives<smiles>[R1]N[C@@H]1[C@H](O)O[C@H](CNS(=O)(=O)c2cccc(Cl)c2Cl)[C@H](O)[C@@H]1O</smiles>

\begin{tabular}{|c|c|c|c|}
\hline \multirow{2}{*}{$\#$} & \multirow{2}{*}{$\mathbf{R} 1$} & \multicolumn{2}{|c|}{ Enzyme $I_{50}(\mu M)^{a}$} \\
\hline & & HK2 & HK1 \\
\hline 31 & & 0.040 & 2.0 \\
\hline 32 & & 0.13 & 10 \\
\hline 33 & & 0.025 & 5.0 \\
\hline 34 & & 0.0079 & 1.0 \\
\hline 35 & & 0.050 & $>\mathbf{2 0}$ \\
\hline 36 & & 0.13 & 63 \\
\hline
\end{tabular}

${ }^{a} \mathrm{IC}_{50}$ values given are means of at least 2 experiments.

conformation change induced by 2,6-disubstituted glucosamines.

To our surprise, some 2,6-disubstituted glucosamines, although shown to be mutually exclusive (i.e., competitive) with G6P in double inhibition mechanistic experiments (Supporting Information), do not extend into the G6P pocket. Instead, the 6-position substituents point into the opening of the catalytic pocket to form a "folded" conformation, exemplified by the cocrystal structure of compound 27 in $\mathrm{HK} 2{ }^{18}$ shown in Figure $2 \mathrm{C}$. In addition to common H-bond interactions with Glu742 and Glu708, the cyano group of compound 27 forms a H-bond with Lys621. The overlay of the cocrystal structures of compound 27 and $\mathbf{3 0}$ in HK2 (Figure 2D) shows minimal change in the protein structures, and the catalytic pocket can accommodate both extended and folded conformations of 2,6-disubstituted glucosamines. This also explains the mutual exclusivity of "folded" compounds with G6P, since the loop (aa533-538, salmon and red loops on the left in Figure 2B) that interacts with G6P is "pushed" open, thereby preventing G6P from binding within the pocket.

To further establish that glycolysis is inhibited by an HK2 inhibitor in a tumor cell line, the following three experiments (details in Supporting Information) were conducted in UMUC-3 cells, a bladder tumor cell line with high protein expression of HK2 and minimal expression of HK1 (Western blot, Supporting Information Figure 6): (1) inhibition of G6P production using a more stable 2-fluoro-2-deoxy-gluocose (2FDG) as a surrogate for glucose; (2) reduction of glucose consumption; and (3) reduction of downstream metabolite lactate (Supporting Information Figure 7A-C). Compound 25 demonstrated inhibition of glycolysis: in all three experiments with an $\mathrm{IC}_{50}$ value of $0.20 \mu \mathrm{M}$ in the first experiment. ${ }^{19}$ Similarly, the selective compounds 33 and 36 had $\mathrm{IC}_{50}$ values of 0.47 and $0.52 \mu \mathrm{M}$, respectively, in the first experiment. Since $\mathrm{HK} 2$ is the dominat isozyme in this cell line, these results 

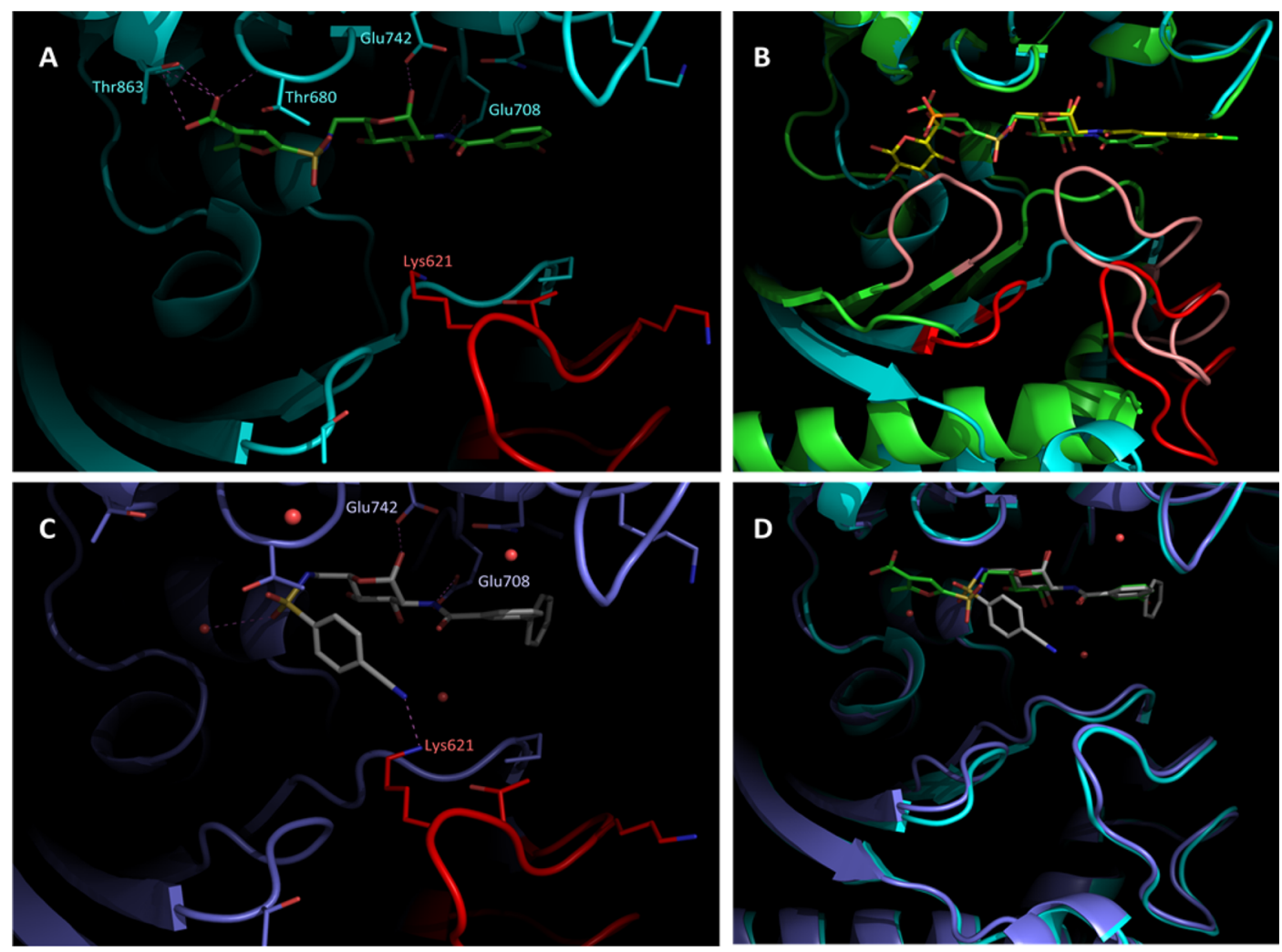

Figure 2. (A) Compound 30 ligand interactions in HK2 in the C-terminal catalytic pocket. (B) Overlay of compound 30 and 1 cocrystal structures with HK2 in the C-terminal catalytic pocket. The salmon and red loops on the left are aa533-538. The salmon and red loops on the right are aa617-633. (C) Compound 27 ligand binding interactions in HK2 in the C-terminal site. (D) Overlay of compounds 27 and 30 in HK2 in the Cterminal catalytic pocket.

provide evidence for HK2 driven cellular activities of this novel 2,6-disubstituted glucosamine series. Although the glucose pockets are likely very different, inhibition of glucose transporters cannot be ruled out in these experiments and may require further investigation. ${ }^{20}$

In summary, we have successfully optimized HK2 HTS hit compound 1 to inhibitors with $\mathrm{nM}$ potency and developed a tractable SAR to achieve a high level of HK2 versus HK1 selectivity. The 2,6-disubstituted glucosamine series was rationally designed based on a cocrystal structure of compound 1 and G6P (Figure 1) to "link" the glucose and G6P binding pockets. A 6-sulfonamide linker turned out to be optimal. The hypothesis of using an acid (compound 30) to mimic the phosphate of G6P was confirmed by the cocrystal structure of compound 30 with HK2 (Figure 2). We also discovered a tractable SAR via the combination of several features that lead to selective HK2 inhibitors over HK1. Several cocrystal structures of HK2 with inhibitors captured different conformations of the enzyme. These cocrystal structures reveal the flexibility of the HK2 protein and that the catalytic site can adopt an "induced-fit" conformation with inhibitors. Using compound $\mathbf{2 5}$ as an example in a tumor cell line such as UMUC-3 that predominately expresses HK2, we have demonstrated that a potent $\mathrm{HK} 2$ small molecule inhibitor can inhibit glycolysis in tumor cells. To our best knowledge, compounds such as $\mathbf{3 3}, \mathbf{3 4}$, and $\mathbf{3 5}$ are the most advanced tool molecules to date in terms of potency and selectivity of HK2 over HK1 to study the role of $\mathrm{HK} 2$ enzymatic activity in tumorigenesis without compromising systemic homeostasis or normal metabolic functions of HK1.

\section{ASSOCIATED CONTENT}

\section{S Supporting Information}

The Supporting Information is available free of charge on the ACS Publications website at DOI: 10.1021/acsmedchemlett.5b00214.

Biological assays, biological data, and experimental procedures, and additional SAR. (PDF)

Data for 17 (PDF)

Data for 32 (PDF)

\section{AUTHOR INFORMATION}

\section{Corresponding Author}

*E-mail: hong.2.lin@gsk.com.

\section{Notes}

The authors declare no competing financial interest.

\section{ACKNOWLEDGMENTS}

The authors thank Dr. Minghui Wang for NMR characterizations of these compounds.

\section{REFERENCES}

(1) Pelicano, H.; Martin, D. S.; Xu, R.-H.; Huang, P. Glycolysis inhibition for anticancer treatment. Oncogene 2006, 25, 4633-4646.

(2) Mathupala, S. P.; Ko, Y. H.; Pedersen, P. L. Hexokinase-2 bound to mitochondria: Cancer's stygian link to the "Warburg effect" and a pivotal target for effective therapy. Semin. Cancer Biol. 2009, 19, 1724

(3) Mathupala, S. P.; Rempel, A.; Pedersen, P. L. Aberrant Glycolytic Metabolism of Cancer Cells: A Remarkable Coordination of Genetic, Transcriptional, Post-translational, and Mutational Events That Lead 
to a Critical Role for Type II Hexokinase. J. Bioenerg. Biomembr. 1997, 29, 339-343.

(4) Pedersen, P. L.; Mathupala, S.; Rempel, A.; Geschwind, J. F.; Ko, Y. H. Mitochondrial bound type II hexohinase: a key player in the growth and survival of many cancers and an ideal prospect for the therapeutic intervention. Biochim. Biophys. Acta, Bioenerg. 2002, 1555, 14.

(5) Ahn, K. J.; Hwang, H. S.; Park, J. H.; Bang, S. H.; Kang, W. J.; Yun, M.; Lee, J. D. Evaluation of the Role of hexokinase type II incellular proliferation and apoptosis using human hepatocellular carcinoma cell lines. J. Nucl. Med. 2009, 50 (9), 1525-1532.

(6) Peng, Q.; Zhou, Q.; Zhou, J.; Zhong, D.; Pan, F.; Liang, H. Stable RNA interference of hexokinase II gene inhibits human colon cancer LoVo cell growth in vitro and in vivo. Cancer Biol. Ther. 2008, 7 (7), $1128-1135$.

(7) Patra, K. C.; Wang, Q.; Bhaskar, P. T.; Miller, L.; Wang, Z.; Wheaton, W.; Chandel, N.; Laakso, M.; Muller, W. J.; Allen, E. L.; Jha, A. K.; Smolen, G. A.; Clasquin, M. F.; Robey, R. B.; Hay, N. Hexokinase 2 Is Required for Tumor Initiation and Maintenance and Its Systemic Deletion Is Therapeutic in Mouse Models of Cancer. Cancer Cell 2013, 24, 213-228.

(8) Lin, H.; Luengo, J. I.; Schulz, M.; Xie, R.; Zeng, J. PCT Int. Appl. (2012), WO2012083145A1, Example 1.

(9) Mulichak, A. M.; Wilson, J. E.; Padmanabhan, K.; Garavito, R. M. The mammalian hexokinase-1. Nat. Struct. Biol. 1998, 5, 555-560.

(10) Kocienski, P.; Pant, C. A convenient preparation of some 2,3,4,6-tetra-O-acetyl-1,5-anhydro-D-hexitols. Carbohydr. Res. 1982, 110 (2), 330-332.

(11) Pdb code 5HGI.

(12) DOI: $10.2210 / \mathrm{pdb} 2 \mathrm{nzt} / \mathrm{pdb}$.

(13) Madsen, R.; Roberts, C.; Fraser-Reid, B. The Pent-4-enoyl Group: A Novel Amine-Protecting Group That is Readily Cleaved under Mild Conditions. J. Org. Chem. 1995, 60, 7920-7926.

(14) Ref 8, intermediate 1.

(15) Ref 8 , intermediate 6a

(16) Deeb, S. S.; Malkki, M.; Laakso, M. Human Hexokinase II: Sequence and Homology to Other Hexokinases. Biochem. Biophys. Res. Commun. 1993, 197 (1), 68-74.

(17) Pdb code 5HEX.

(18) Pdb code 5HFU.

(19) Although not selective for HK2 vs HK1, compound 25 is a very weak inhibitor of $\mathrm{HK} 4$ (glucokinase $\mathrm{IC}_{50}=3.5 \mu \mathrm{M} ; 120$-fold selective).

(20) Deng, D.; Xu, C.; Sun, P.; Wu, J.; Yan, C.; Hu, M.; Yan, N. Crystal structure of the human glucose transporter GLUT1. Nature 2014, 510, 121-125. 